Relations industrielles

Industrial Relations

\title{
Les relations du travail dans la fonction publique au Québec : situation et orientation Public Service Staff Relations in Quebec
}

\section{Gérard Hébert}

Volume 29, numéro 4, 1974

URI : https://id.erudit.org/iderudit/028553ar

DOI : https://doi.org/10.7202/028553ar

Aller au sommaire du numéro

Éditeur(s)

Département des relations industrielles de l'Université Laval

ISSN

0034-379X (imprimé)

1703-8138 (numérique)

Découvrir la revue

Citer cet article

Hébert, G. (1974). Les relations du travail dans la fonction publique au Québec : situation et orientation. Relations industrielles / Industrial Relations, 29(4),

750-775. https://doi.org/10.7202/028553ar
Résumé de l'article

L'auteur résume les derniers développements survenus dans le secteur public québécois depuis la grève générale de 1972. Après avoir effectué une brève rétrospective des incidents rencontrés dans cette négociation, il décrit les principales orientations qui se dégagent tant du point de vue des structures de négociation que de celui des mécanismes de règlement des conflits en vue de la prochaine ronde de négociation.
Tous droits réservés (C) Département des relations industrielles de l'Universite Laval, 1974
Ce document est protégé par la loi sur le droit d'auteur. L'utilisation des services d'Érudit (y compris la reproduction) est assujettie à sa politique d'utilisation que vous pouvez consulter en ligne. 


\section{Les relations du travail dans la fonction publique au Québec}

\section{Situation ef orientations}

\section{Gérard HÉBERT}

L'auteur résume les derniers développements survenus dans le secteur public québécois depuis la grève générale de 1972. Après avoir effectué une brève rétrospective des incidents rencontrés dans cette négociation, il décrit les principales orientations qui se dégagent tant du point de vue des structures de négociation que de celui des mécanismes de règlement des conflits en vue de la prochaine ronde de négociation.

Alors que dans plusieurs provinces canadiennes les lois sur les relations de travail dans la fonction publique ont subi d'importantes modifications au cours des toutes dernières années, celles du Québec n'ont connu aucun développement spectaculaire ${ }^{1}$. Toutes les conventions collectives visant les employés des secteurs public et parapublic au Québec - sauf une ou deux exceptions - sont en vigueur depuis près de deux ans et le demeureront jusqu'au 30 juin 1975. Elles ont été signées, pour la plupart, à l'automne de 1972, à la suite de longues et difficiles négociations entre le Gouvernement du Québec et le front commun des centrales syndicales C.S.N. - F.T.Q. - C.E.Q.

Après un tel affrontement, qui a déjà fait l'objet de plusieurs études $^{2}$, une période d'accalmie s'imposait. Elle n'est cependant pas sans intérêt. Quelques développements y ont pris place qui pourraient avoir une influence décisive sur certains aspects importants des futures négociations. Nous verrons particulièrement ce qui a trait aux structu-

HÉBERT, G., professeur titulaire, Ecole des relations industrielles, Université de Montréal, Montréal.

$1 \mathrm{La}$ présente communication a été donnée au Congrès de l'Institut canadien de recherches en relations industrielles, à l'Université de Toronto, le 3 juin 1974. Le texte final a été rédigé en septembre 1974. 
res de négociation, au règlement des conflits et au champ d'application de la convention collective. Rappelons que les fonctionnaires sont, au Québec, régis par le Code du travail, comme tous les autres salariés, sauf sur certains points que nous relèverons au cours de l'exposé.

\section{STURCTURES DE NÉGOCIATION}

Les structures de négociation du secteur public n'ont pas été modifiées substantiellement depuis 1967 ; mais quelques événements, dans les domaines syndical et juridique, méritent d'être relevés : ils peuvent influencer les structures à venir.

\section{Syndicats et unités de négociation}

Dans la fonction publique du Québec, on compte huit unités de négociation, d'importance très différente. Le syndicat des fonctionnaires provinciaux du Québec (S.F.P.Q.) compte, à lui seul, plus de $75 \%$ de tous les fonctionnaires syndiqués : la Loi de la fonction publique l'a désigné pour représenter tous les salariés directs de l'Etat - sauf quelques exceptions - selon deux groupes distincts, les fonctionnaires proprement dits et les ouvriers. La loi reconnaissait en même temps quatre autres groupes, beaucoup moins nombreux et assez bien caractérisés : les enseignants à l'emploi du gouvernement, les professionnels membres des professions traditionnelles, les «nouveaux 》 professionnels et les agents de la paix (gardiens de prison, garde-chasse, inspecteurs des autoroutes et autres employés de même type $)^{3}$. En fait, on retrouve huit unités de négociation distinctes comme il apparaît au tableau 1.

Le Syndicat des fonctionnaires provinciaux a été, jusqu'en 1972, affilié à la C.S.N. Par une décision d'un comité composé de 41 membres et mandaté par son conseil provincial, il s'est retiré du front commun le 14 août 1972. Un référendum, tenu le 25 septembre, avait décidé de la désaffiliation, mais à une très faible majorité des voix. La division

2 Jean BOIVIN, «La négociation collective dans le secteur public québécois : une évaluation des trois premières rondes (1964-1972) , Relations industrielles, 27, 4 (octobre 1972), pp. 691-708. Shirley GOLDENBERG, «Dispute Settlement in the Public Sector: The Canadian Scene », dans Industrial Relations Research Association, Proceedings of the Twenty-Fifth Anniversary Meeting, December 28-29, Toronto; Madison, Wis., I.R.R.A., c. 1973, pp. 58-59. Gérard HÉBERT, « La négociation du secteur public et parapublic au Québec, 1971-1972 », La Gazette du travail, 73, 1 (janvier 1973), pp. 26-35. Mme GOLDENBERG prépare une étude d'envergure sur la négociation dans le secteur public au Québec.

3 Loi de la fonction publique, art. 69-70. 
sur ce point demeure profonde parmi les membres, et personne ne saurait prédire le résultat d'un nouveau référendum, si on le tenait à l'heure actuelle. Pour l'instant, cependant, c'est le statu quo, et le S.F.P.Q. demeure toujours absent des tentatives de regroupement du front commun, par exemple de celui qu'on a tenté à l'occasion des demandes de réouverture des conventions collectives par suite de l'augmentation considérable du coût de la vie ${ }^{4}$.

On retrouve une hésitation semblable chez les agents de la paix. Un certain nombre d'entre eux - peut-être pour des raisons strictement financières, comme bon nombre de fonctionnaires - remettent en cause le contrat de service avec la C.S.N. Le malaise est assez sérieux pour qu'on veuille tenir un référendum lorsque les négociations avec le gouvernement seront terminées, soit vers la fin de l'année 1974.

\section{TABLEAU 1}

\section{Les unités de négociation dans la fonction publique du Québec}

Groupe principal

Syndicat des fonctionnaires provinciaux du Québec

Unité «fonctionnaires »

Unité « ouvriers》

22,000

11,000

Professionnels

Syndicat des professionnels du gouvernement du Québec (CSN) 1 3,200

Syndicat professionnel des comptables agréés du gouvernement du Québec

Syndicat des avocats et notaires de la fonction publique

Syndicat professionnel des médecins du gouvernement

$\left.\begin{array}{c}\text { du Québec } \\ \text { Association professionnelle des chirurgiens-dentistes du } \\ \text { gouvernement du Québec }\end{array}\right\}$

\section{Enseignants}

Syndicat des professeurs de l'Etat du Québec 2 (CSN)

Agents de la paix

Syndicat des agents de la paix de la fonction publique 3

4 La Presse, jeudi le 6 juin 1974, p. A 6.

1 Ce syndicat réunit 22 groupes de professionnels différents; ces divers groupes, cependant, ne constituent qu'une seule unité de négociation.

$2 \mathrm{Ce}$ syndicat appartient à la Fédération nationale des enseignants du Québec (F.N.E.Q.) affiliée à la C.S.N.

3 Le syndicat des agents de la paix ne peut être affilié à aucune centrale (Loi de la fonction publique, art. 74), mais il possède depuis ses débuts une entente de service avec la C.S.N.; il a eu, très longtemps, ses bureaux dans l'édifice de la C.S.N. à Montréal.

SOURCES : Québec, ministère de la Fonction publique, Rapport annuel 197273 , pp. 33-34; Rapport annuel 1970-71, p. 64. Portrait des secteurs public et parapublic, les effectifs et les salaires (28 février 1972), pp. 69-72. Gérard Hébert, loc. cit., pp. 34-35. 
D'un autre côté, les enseignants à l'emploi du gouvernement demeurent fermes dans leur affiliation à la F.N.E.Q., une des fédérations les plus militantes de la C.S.N. Le syndicat interprofessionnel est également affilié à la C.S.N., alors que les syndicats séparés des anciennes professions constituent autant de syndicats indépendants.

Pour compléter le tableau, il faudrait ajouter que le gouvernement a reconnu, d'une certaine façon, le Syndicat des cadres du gouvernement du Québec et la Fraternité des cadres intermédiaires des agents de la paix. Il a, entre autres, autorisé le prélèvement de la cotisation de ces deux associations, moyennant une demande expresse de leurs membres, formulée individuellement par chacun ${ }^{5}$. Pour ces cadres syndiqués, le Conseil du Trésor a émis une «Directive concernant une procédure de règlement de griefs »; en sont exclus les seuls adjoints aux cadres supérieurs et les cadres supérieurs eux-mêmes ${ }^{6}$.

On n'inclut pas dans la fonction publique proprement dite les employés de la Société des alcools du Québec, de Radio-Québec et d'autres organismes du même genre. Il faut noter cependant que le ministère de la Fonction publique a juridiction pour négocier les conventions collectives de ces employés tout comme celles des fonctionnaires proprement dits. Il le fait par l'entremise de sa Direction générale des relations du travail (D.G.R.T.). Le gouvernement effectue aussi des négociations avec d'autres groupes; ainsi, la Régie de l'assurance-maladie du Québec négocie avec les médecins les tarifs que ceux-ci peuvent exiger en vertu du régime d'assurance-maladie. Mais ces diverses négociations n'ont rien à voir, directement, avec les négociations de la fonction publique.

\section{Précisions aux accréditations}

La Loi de la fonction publique, adoptée le 5 août 1965, a déterminé les principales unités de négociation. Elle a reconnu nommément le S.F.P.Q. comme représentant des fonctionnaires et des ouvriers salariés du gouvernement (art. 69-70). Elle a statué, en même temps, pour les autres groupes, que l'accréditation serait accordée par le lieutenant-gouverneur en conseil, sur la recommandation d'un comité conjoint, constitué à cette fin par le lieutenant-gouverneur en conseil lui-même. Les accréditations

5 Québec, ministère de la Fonction publique, Rapport annuel 1972-73, p. 45.

6 Gouvernement du Québec, Conseil du Trésor, CT 65,044, 12 juillet 1972. 
mentionnées plus haut ont été accordées de cette façon, à l'exception du S.F.P.Q. qui jouit d'une accréditation statutaire.

Depuis lors, les précisions aux unités d'accréditation ont été formulées, conformément à la loi, par la Commission des relations de travail et, depuis 1969, par le Tribunal du travail (art. 70-71). C'est ainsi, par exemple, que la Commission des relations de travail a décidé que les cuisiniers des prisons et des maisons de détention, réclamés par le Syndicat des agents de la paix, appartenaient au S.F.P.Q., unité des ouvriers ${ }^{7}$.

Un cas plus important, à cause du principe qu'il impliquait, visait les secrétaires des juges de la Cour supérieure. Le gouvernement voulait les faire exclure de l'unité de négociation à cause du caractère confidentiel de leurs fonctions. L'article 76 de la Loi de la fonction publique avait justement modifié la définition du salarié, selon le Code du travail, pour en exclure « un fonctionnaire du gouvernement dont l'emploi est... d'un caractère confidentiel ». Le Tribunal du travail a rejeté l'argument, en soulignant que l'exclusion ne vise que la confidentialité en matière de relations de travail.

Le cas le plus important n'est pas encore définitivement tranché. Le 20 mars 1973, le S.F.P.Q. déposait une requête en accréditation pour représenter les employés occasionnels du gouvernement, fonctionnaires et ouvriers. Le syndicat avait fait signer plus de 1,000 cartes à des ouvriers de ce genre ${ }^{8}$. Devant le commissaire-enquêteur, le gouvernement soutint, en septembre 1973, que celui-ci n'avait pas juridiction pour entendre la demande puisque l'article 3 de la Loi de la fonction publique accorde à la Commission de la fonction publique le pouvoir de soustraire les employés occasionnels à l'application de cette loi et le droit de déterminer par règlement la manière dont leurs emplois seront régis. Dans sa décision du 22 janvier 1974, le commissaire-enquêteur fit droit à l'objection ${ }^{9}$. Le syndicat en appela au Tribunal du travail; celui-ci déclara, dans un jugement rendu le 5 avril, que l'article 3 de la Loi de la fonction publique n'avait pour effet que de soustraire les occasionnels à l'application de la Loi de la fonction publique. Selon le Tribunal, cet article n'enlève pas

7 Syndicat des agents de la paix vs SFPQ, 15 mai 1968, juge Georges Chassé, Commission des relations de travail, dossier no 11517, cas no 1573 .

8 Journal S.F.P.Q., 13, 47 (1er avril 1974), p. 10, 13, 48 (20 avril 1974), p. 7.

9 Québec, Jurisprudence en droit du travail, Décisions des Commissaires-enquêteurs, 6, 1 (janv.-fév. 1974), pp. 47-51. 
aux fonctionnaires, même occasionnels, leur statut de salarié et leur droit de se prévaloir des dispositions du Code du travail ${ }^{10}$.

Vu l'importance de l'enjeu, le gouvernement a présenté, devant la Cour supérieure, une requête pour l'émission d'un bref d'évocation et de revision de ce jugement. La Cour supérieure a rejeté la requête au cours de l'été de $1974{ }^{11}$. Conformément à la décision du Tribunal du travail, le dossier a été retourné au commissaire-enquêteur pour que celui-ci termine l'enquête qu'il n'avait pas effectuée, parce qu'il considérait alors qu'il n'avait pas juridiction.

L'inclusion éventuelle des employés occasionnels de l'Etat dans l'unité de négociation pourrait avoir d'intéressantes implications. Leur exclusion accordait jusqu'ici aux différents ministères une souplesse d'action très utile. Par contre, l'absence de contrôle rigoureux par la Commission de la fonction publique ouvrait la porte à bien des abus, semble-t-il, tant en ce qui a trait aux influences politiques qu'aux conditions de travail ellesmêmes. L'activité syndicale à leur endroit pourrait amener plusieurs redressements.

\section{Situation des conventions collectives}

La convention collective du S.F.P.Q. échoit le 30 juin 1975. Il en va de même pour toutes les grandes conventions collectives du secteur parapublic, ainsi que pour la plupart des autres conventions de la fonction publique. Seuls les agents de la paix font exception : leur convention collective échéait le 30 juin 1974; ils ont négocié depuis le printemps et une entente de principe est intervenue à la fin de septembre; elle doit être ratifiée par les membres ${ }^{12}$.

La convention collective des fonctionnaires a subi certaines modifications depuis son adoption en 1972, grâce, entre autres, à l'activité du comité de relations professionnelles établi en vertu de l'article 10 de la convention. Sans qu'il s'agisse de négociations proprement dites, mais plutôt de consultations, l'activité du comité a permis de modifier la con-

10 Syndicat des fonctionnaires provinciaux du Québec v. Gouvernement $d u$ Québec, 5 avril 1974. Juge Gaston Michaud. Tribunal du travail, nos 74-685 et 74686.

11 Procureur général de la province de Québec v. Tribunal du travail et al. 17 juin 1974. Juge Beaudoin. Cour supérieure du Québec. Rapporté dans $\mathrm{CCH}$, Canadian Labour Law Reports, no 14245. Le gouvernement a interjeté appel de la décision de la Cour supérieure; l'affaire demeure donc en suspens.

12 L'Agent de la paix, vol. 8, nos 6 à 8 (juin, juillet, août 1974). 
vention sur plusieurs points. Ainsi, on a pu régler le cas des congés de maladie et améliorer considérablement le régime des frais de voyage. Sur ce dernier point, en particulier, à cause de la campagne de publicité, des alliances effectuées avec d'autres syndicats et des actions entreprises, la modification a revêtu un peu l'allure d'une négociation ${ }^{13}$.

Cependant, la véritable tentative de réouverture s'est faite sur le problème de l'augmentation du coût de la vie. La convention collective prévoyait le paiement d'un montant forfaitaire calculé selon la variation de l'indice des prix à la consommation, plus précisément selon l'excédent de cet indice par rapport à un pourcentage déterminé d'avance (art. 29.08). Toutes les conventions collectives du secteur public et parapublic contiennent une disposition semblable. Aussi, le front commun C.S.N. F.T.Q. - C.E.Q. a-t-il tenté de faire ouvrir toutes les conventions, demandant que l'augmentation prévue soit transférée à l'échelle de salaire plutôt que d'être payée en montant forfaitaire. Le S.F.P.Q. a tenté, de son côté, d'obtenir un règlement analogue. En fait, le gouvernernent a refusé de rouvrir la convention; il a voulu s'en tenir aux dispositions de l'article en question. Il a cependant accepté d'avancer le paiement d'une partie du montant forfaitaire: il a versé en juin un peu plus de la moitié du montant probable, et il devait payer la balance à l'automne, au moment où le paiement total devait se faire en vertu de la convention elle-même ${ }^{14}$. On peut supposer, à moins que le rythme de l'inflation ne diminue considérablement, que l'indexation pure et simple fera l'objet d'une demande particulièrement intense lors des prochaines négociations.

\section{Structures des prochaines négociations}

On ne peut faire plus, présentement, que de spéculer sur les structures des prochaines négociations. Du point de vue légal, la Loi $46^{15}$ est toujours en vigueur. Elle prévoit une douzaine de tables de négociation, formées en tenant compte des trois facteurs suivants : l'affiliation syndicale (C.S.N., F.T.Q., C.E.Q., et autres), les catégories de salariés (enseignants, non-enseignants) et les secteurs principaux (commissions scolaires, C.E. G.E.P., et hôpitaux). D'un autre côté, la Loi 46 cessera d'exister à

13 Journal S.F.P.Q., 13, 45 (24 janvier 1974), numéro entier; 13, 46 (8 mars 1974), numéro entier; 13, 38 (20 avril 1974) pp. 2 et 6.

14 La Presse, mercredi 29 mai 1974, p. A 9 et jeudi 6 juin 1974, p. A 6.

15 Loi du régime de négociations collectives dans les secteurs de l'éducation et des hôpitaux. Lois du Québec, 1971. 
l'expiration des conventions collectives présentement en vigueur. Il faudra donc en adopter une nouvelle au cours de l'année qui vient.

Si l'on en juge par les prises de position des différentes fédérations ou centrales impliquées, on ne semble pas s'orienter, au moment présent, vers un front commun de tous les salariés du secteur public et parapublic, mais plutôt vers un regroupement par secteur : la fonction publique, l'éducation, les affaires sociales ${ }^{16}$. Même au niveau de chacun de ces secteurs, la collaboration intersyndicale n'est pas assurée; elle pourra d'ailleurs varier sensiblement d'un secteur à l'autre. Les affrontements des deux principales centrales dans le monde de la construction ne faciliteront pas les choses.

Cependant, absence de front commun ne signifie pas absence de coordination. Les divers groupes parlent déjà de «contacts». Chacun veut demeurer autonome, pour mieux assurer la défense de ses propres intérêts et la recherche de solutions à ses problèmes particuliers; par contre, on voudra sûrement s'aider, et la forme minimale d'une telle collaboration - qui peut cependant être très efficace - paraît être celle des négociations coordonnées.

S'il y avait centralisation plus grande, elle pourrait bien venir de la partie patronale. La loi du ministère de la fonction publique, sanctionnée le 28 novembre 1969, lui confie la charge de négocier toutes les conventions collectives auxquelles le gouvernement est partie (art. 2). A cette fin, le ministère a établi une Direction générale des relations de travail qui prépare présentement les futures négociations, conjointement avec les partenaires officiels du gouvernement, c'est-à-dire les commissions scolaires et les administrations hospitalières, par l'entremise de leurs organisations provinciales respectives. Déjà on prévoit, en plus des trois groupes principaux (la fonction publique, l'éducation et les affaires sociales), un quatrième groupe que l'on commence à appeler le secteur péripublic, qui renfermerait des organismes comme Radio-Québec, la Société des alcools du Québec et quelques autres organismes semblables. Pour des raisons de simplification administrative, la centralisation aura toujours un attrait particulier pour le gouvernement. Par contre, à cause des degrés différents auxquels il se trouve impliqué dans ces différents secteurs, il ne faut pas écarter la possibilité que le gouvernement veuille s'orienter vers une négociation à plusieurs niveaux : on pourrait négocier à une table centrale les questions très générales, comme une norme globale

16 La Presse, jeudi 23 mai 1974, p. A 10 et vendredi 27 septembre 1974, p. A 5. 
d'augmentation de salaire, et en renvoyer toutes les applications aux tables sectorielles. Il reste que les représentants du gouvernement sont, en ce moment, très peu loquaces sur les projets et les préparatifs des prochaines négociations.

\section{IMPASSES ET DROIT DE GRÈVE}

La question du droit de grève dans le secteur public retient l'attention générale plus que le problème des structures de négociation ou du champ d'application de la convention. À cause des structures introduites en 1971-1972, au Québec, on ne limite pratiquement jamais cette discussion à la seule fonction publique; on y englobe toujours le secteur parapublic. Depuis deux ans, l'attention s'est surtout portée sur les services essentiels. Un projet de loi a été présenté en décembre 1972, mais, sous l'abondance des critiques, il a été finalement retiré. L'événement peut cependant révéler certaines orientations. Lois et projets de lois feront donc l'objet principal de cette partie.

\section{Lois existantes}

La Loi de la fonction publique ne contient que deux prescriptions en matière de règlement des différends. Au sujet des services essentiels, elle stipule que la grève est interdite à tout groupe de salariés du gouvernement, à moins que les services essentiels et la façon de les maintenir n'aient été déterminés par entente préalable entre les parties ou par décision du Tribunal du travail. De plus, la grève est interdite en tout temps aux agents de la paix (art. 75). Les membres de la Sûreté du Québec n'ont pas droit de grève, non plus, mais en vertu d'une loi qui leur est propre. Dans le cas des agents de la paix, aucun mécanisme n'est prévu dans la loi pour le règlement final des conflits qui les impliquent. La convention collective y a suppléé partiellement : un article stipule que, faute d'entente sur les échelles de traitement, le différend doit être soumis, à la demande d'une des parties, à un arbitre choisi par les parties ou nommé par le ministre du Travail; la sentence de l'arbitre, toutefois, ne constitue qu'une recommandation au lieutenant-gouverneur en conseil ${ }^{17}$. De fait, à l'été de 1973, le Syndicat des agents de la paix demanda l'arbitrage, et les recommandations de l'arbitre furent incorporées au reste de la convention par l'arrêté-en-conseil no 204 du 16 janvier $1974{ }^{18}$.

17 Convention collective intervenue entre le Syndicat des agents de la paix et le gouvernement du Québec, le 31 août 1973, art. 46.01 et 46.04.

18 Voir L'Agent de la paix, 8, 1 (janvier 1974), p. 1. 
En dehors de ces cas particuliers, l'exercice du droit de grève dans tout le secteur public et parapublic est régi, depuis 1964, par l'article $99 \mathrm{du}$ Code du travail. Cet article ajoute aux exigences de conciliation et de délai, applicables à tous les cas de conflits, certaines dispositions supplémentaires. Ainsi, s'il s'agit d'un service public, le syndicat doit donner avis au ministre, au moins huit jours à l'avance, de la date à laquelle il entend recourir à la grève. Le Cabinet des ministres peut décider alors s'il juge qu'un arrêt de travail dans ce service mettrait la santé ou la sécurité publique, ou l'éducation, en danger; si oui, il institue une commission d'enquête, après quoi le procureur général peut demander à un juge de la Cour supérieure d'émettre une injonction pour suspendre la grève durant le cours de l'enquête; l'injonction peut être prolongée jusqu'à vingt jours après l'expiration du délai de soixante jours accordé à la commission d'enquête, mais jamais au-delà. À ce moment, si le conflit n'est pas réglé, et s'il y a urgence publique, le seul recours demeure la convocation de l'Assemblée nationale et l'adoption d'une loi spéciale.

Ce sont là les dispositions qui s'appliquaient lors de la grève du front commun en 1972. Après avoir utilisé l'injonction sous l'article 99 dans les cas les plus pressants - l'Hydro-Québec, les hôpitaux psychiatriques et les institutions pour malades chroniques - le gouvernement mit fin à l'arrêt de travail dans l'ensemble des hôpitaux et des écoles par une loi spéciale, adoptée et sanctionnée le 21 avril 1972. En plus d'ordonner le retour au travail pour le jour suivant (art. 2) et de suspendre le droit de grève jusqu'au 30 juin (art. 6), la Loi 19 ordonnait la convocation de la Commission parlementaire de la fonction publique pour le 25 avril (art. 8). La loi envisageait aussi la possibilité qu'une entente collective soit conclue avant le 1er juin 1972; autrement, le gouvernement devrait déterminer par décret, au plus tard le 30 juin 1972, les conditions de travail des salariés jusqu'au 30 juin 1974 (art. 10).

La période de temps allouée s'avérant insuffisante, l'Assemblée nationale dut adopter une autre loi, la Loi 53. Celle-ci prolongeait la suspension du droit de grève jusqu'à ce que les conditions de travail de l'ensemble des salariés aient été établies suivant la loi. Elle visait surtout à favoriser le plus possible la conclusion d'ententes par voie de négociation : les négociations pouvaient se poursuivre jusqu'au 15 septembre 1972, et même au delà, si les parties s'entendaient pour les poursuivre davantage; c'est seulement en cas d'échec de ces négociations que le gouvernement imposerait les conditions de travail par décret. Toutes les conventions furent ainsi négociées, sauf celle des enseignants et des employés de la 
Société des Alcools du Québec, pour qui le gouvernement adopta un décret, en décembre 1972, conformément aux prescriptions de la loi.

Malgré l'ampleur des difficultés rencontrées, malgré la menace de grève généralisée et malgré les séquelles des actions judiciaires, y compris l'emprisonnement des chefs syndicaux, il faut constater que le gouvernement a fait des efforts considérables pour régler par voie de discussion et d'entente mutuelle les différends qui existaient aux nombreuses tables de négociation. Même dans le cas du décret des enseignants, le gouvernement ne pouvait procéder, en vertu même des termes de la loi, qu'après consultation des syndicats impliqués et des employeurs intéressés. Car le gouvernement n'était pas seul à négocier avec les syndicats : les représentants des commissions scolaires et des administrations hospitalières constituaient les employeurs véritables, aux termes de la loi et, à ce titre, ils étaient des partenaires nécessaires du gouvernement; celui-ci devait négocier avec eux tout autant qu'avec les syndicats, bien que sur un autre plan.

\section{Services essentiels}

L'ensemble de l'événement avait mis en relief le problème des services essentiels. Leur maintien n'est assuré, en vertu de la loi, que dans le cas de la fonction publique; le Code du travail n'en fait aucune mention. Le ministre du Travail avait promis, à diverses reprises, d'introduire une législation générale sur le sujet aussitôt que les conventions collectives en cause seraient réglées. Un conflit, en quelque sorte retardé, à l'Hydro-Québec, l'oblige à présenter d'abord une loi particulière.

On se souvient que les employés de l'Hydro-Québec s'étaient dissociés du front commun au moment du déclenchement de la grève illimitée, en avril 1972. Ils avaient poursuivi leur négociation séparément et s'étaient mis en grève au mois de novembre. La grève paraissait sans issue. Aussi, le 15 novembre 1972, l'Assemblée nationale adopta le projet de loi 73; celui-ci ordonnait à tout employé de l'Hydro-Québec dont la fonction visait un service considéré essentiel de rentrer au travail sous peine d'amendes considérables. La loi définissait les services essentiels de trois manières. La première définition consistait en une longue énumération, qui incluait le plein fonctionnement de tous les services d'électricité et de gaz au public, ainsi que toute la production, transformation, transmission et distribution d'électricité, avec les services ancillaires des magasiniers, de la paye, de la sécurité et même du travail de bureau. L'énumération était très longue, sans doute dans l'intention de forcer les parties à 
lui préférer la deuxième ou la troisième définition; dans la seconde définition, la loi reconnaissait comme services essentiels ceux sur lesquels les parties se seraient entendues entre elles; la troisième définition serait celle qui émanerait du Tribunal du travail à la demande d'une des parties.

C'est un mois plus tard, en décembre 1972, que le ministre du Travail introduisit le projet de loi 89 , intitulé «Loi assurant le bien-être de la population en cas de conflit de travail ». Le projet spécifiait que la grève était interdite aux salariés d'un service public avant que les services essentiels et la façon de les maintenir n'aient été déterminés soit par entente préalable entre les parties, soit par le Tribunal du travail à la demande d'une des parties. Cette fois, cependant, le projet de loi était beaucoup plus général. Il instituait divers mécanismes pour tenter de régler les différends susceptibles d'affecter la santé, la sécurité, l'éducation et même le bien-être du public.

Le projet prévoyait d'abord l'institution d'une commission d'enquête, qui devait décider si l'arrêt de travail compromettait vraiment la santé, la sécurité ou le bien-être du public, ou encore l'éducation, dans l'ensemble du Québec ou dans une de ses régions (art. 5-10 et 18). Si la commission répondait affirmativement, le gouvernement pouvait alors interdire la grève, s'il s'agissait d'un service public relevant du gouvernement (art. 11), ou la suspendre pour au plus soixante jours dans tous les autres cas (art. 12-13 et 19). Pendant cette période, le gouvernement pouvait, s'il s'agissait d'un service public relevant de lui, déférer le conflit à la commission parlementaire la plus appropriée : celle-ci devait faire rapport de l'état des négociations et des dernières offres patronales ou gouvernementales; le gouvernement pouvait aussi nommer un médiateur ou prendre toute autre mesure jugée utile pour faciliter la négociation. Quelles que soient les mesures adoptées à cette étape, la loi ordonnait la tenue d'un scrutin secret sur les dernières offres patronales dans les quinze jours suivant la date du rapport de la commission parlementaire; si on acceptait les offres, celles-ci tenaient lieu de convention collective; sionn, le gouvernement pouvait décider de lever l'interdiction de la grève ou de convoquer l'Assemblée nationale pour trancher la question (art. 15-17). Dans les cas de services publics ne relevant pas du gouvernement et, en général, dans le secteur privé, quand la santé, la sécurité, le bien-être public ou l'éducation étaient en cause, le gouvernement pouvait soit procéder de la même manière que dans le cas d'un service public relevant de lui, soit remettre le cas aux commissaires aux différends qui devaient le régler en choisissant intégralement, sans la modifier, l'une des deux dernières offres («last-offer selection $»$ ) (art. 18-22). 
Les objectifs que visait le projet de loi 89 étaient louables : il voulait assurer le maintien des services essentiels durant tout conflit de travail dans les services publics, et instituer en même temps des mécanismes de règlement du conflit dans les cas où la santé, la sécurité, le bien-être et l'éducation du public étaient en cause, soit dans l'ensemble de la province soit dans une de ses régions. Il n'eut cependant pas beaucoup de supporteurs. Même les associations patronales le dénoncèrent comme trop compliqué et donnant trop de pouvoir au Conseil des ministres. Les syndicats, de leur côté, menèrent une campagne acharnée contre le projet; ils soutenaient que c'était la disparition, à toute fin pratique, du droit de grève, à cause des trop grands pouvoirs accordés au gouvernement. En fait, le ministre du Travail, après avoir dit que ce n'était qu'un instrument de travail pour amorcer la discussion, spécialement devant la commission parlementaire, finit par le retirer complètement. On lui avait reproché, entre autres choses, d'avoir soumis ce projet de loi sans avoir demandé l'avis du Conseil consultatif du travail et de la main-d'oeuvre.

En effet, à la fin de 1972, le Conseil consultatif du travail et de la main-d'oeuvre ne s'était pas réuni depuis près d'un an ${ }^{19}$. En fait, le ministre lui-même avouera qu'il ne l'avait pas consulté une seule fois depuis le début de son mandat ${ }^{20}$. Le ministre du Travail soumit donc son projet de loi 89 au Conseil consultatif, en lui demandant de s'attacher davantage à l'ensemble des mécanismes de négociation et de règlement des différends plutôt qu'à la lettre du projet. Il lui suggérait, en même temps, de considérer d'abord les cas plus simples de services publics qui dépendent d'un employeur unique, comme par exemple l'Hydro-Québec ou la Corporation de gaz métropolitain ${ }^{21}$. Les débats se concentrèrent rapidement sur les services essentiels. Il faut bien constater que la force de frappe d'un syndicat, à l'occasion d'une grève dans un service public, consiste précisément dans le caractère essentiel de ce service. Au Conseil, on s'accordait sur la nécessité de déterminer les services essentiels. Mais

19 Conseil consultatif du travail et de la main-d'oeuvre, Troisième rapport annuel 1971-72, pp. 35, 63; Quatrième rapport annuel 1972-73, pp. 11, 16, 24-25. Conseil du patronat du Québec, Bulletin sur les relations du travail, 3, 26 (décembre 1972), p. 2. Les activités du Conseil consultatif étant considérées comme confidentielles, le «monde ordinaire» ne peut en prendre connaissance que par certaines déclarations que veulent bien faire à leur sujet soit les membres du Conseil, soit le ministre du Travail lui-même.

20 Québec, Assemblée nationale, Journal des Débats, Commission parlementaire, 2e Session, 30e Législature, Commission permanente du travail, de da maind'oeuvre et de l'immigration, 6 juin 1974, no 82, p. B-3048.

21 Ibid., 5 juin 1974, no 80, p. B-2992. 
là s'arrêtait le consensus. Dans le cas où les parties ne parviendraient pas à s'entendre sur les services essentiels à maintenir, les représentants syndicaux proposaient l'intervention d'un médiateur, dont les recommandations seraient publiées mais ne posséderaient aucune force coercitive ${ }^{22}$; les représentants patronaux, de leur côté, exigeaient que, faute d'entente, les services essentiels soient déterminés par une tierce partie, soit le Tribunal du travail, soit préférablement, une Régie nouvelle, indépendante du pouvoir politique et dotée de pouvoirs administratifs et judiciaires ${ }^{23}$.

\section{Projets de loi}

Le 12 juillet 1974, le ministre du Travail dépose, en première lecture, le projet de loi 24. Celui-ci contient une longue série d'amendements à de nombreux articles du Code du travail, mais rien sur l'article 99. On peut supposer que le gouvernement n'a pas réussi à obtenir un consensus suffisant parmi les intéressés, et peut-être même au sein de son propre caucus, pour soumettre, sur le sujet, un projet précis. Mais il devra le faire bientôt.

Le ministre du Travail, de son côté, avait déjà fait connaître son opinion, d'abord en réponse à des journalistes, puis devant la Commission parlementaire du travail et de la main-d'oeuvre ${ }^{24}$. Selon lui, le nouvel article 9925 visera d'abord les services publics qui en relèvent que d'un employeur unique, ce qu'il appelle «les entreprises monolithiques »; il ajoute que ses prescriptions ne s'appliqueront pas nécessairement aux services publics qui tombent sous l'emprise actuelle de la loi 46 , les hôpitaux, les écoles et les collèges ${ }^{26}$.

22 Marcel PEPIN, «Aux membres du Conseil consultatif du travail », 30 octobre 1973. Document polycopié, 6 pages. «Projet d'une prise de position F.T.Q. sur la question du maintien des services essentiels $\gg$. Texte polycopié, sans date, 3 pages.

23 Ghislain DUFOUR, «Une autre tangente malheureuse de M. Jean Cournoyer $\gg$, Le Devoir, samedi le 13 juillet 1974, p. 5.

24 La Presse, jeudi 16 mai 1974, p. A 12. Québec, Assemblée nationale, Journal des Débats, Commission permanente du travail, de la main-d'oeuvre et de l'immigration, 5 juin 1974, no 80 , pp. B-2990 à B-2994 et 6 juin 1974, no 82, pp. B3041 à B-3048.

25 Le texte du nouvel article 99, tel que soumis pour consultation au Conseil consultatif du travail et de la main-d'oeuvre, a été publié dans C.P.Q., Bulletin sur les relations du travail, 5, 41 (avril 1974), p. 2.

26 Québec, Assemblée nationale, Journal des Débats, Commission permanente du travail, de la main-d'oeuvre et de l'immigration, 5 juin 1974, p. B-2992. 
Alors que le projet de loi 24 prévoit de rendre la conciliation facultative et de séparer ainsi l'exercice du droit de grève de ce recours éventuel, il n'en irait pas de même dans les services publics. Toujours selon le projet d'article 99, en cas d'échec des négociations directes, l'une ou l'autre des parties en donne avis au ministre, qui constitue un conseil de conciliation tripartite, formé de la manière habituelle: un membre désigné par la partie syndicale, un autre par la partie patronale et un président choisi par les deux premiers, ou par un comité de trois juges du Tribunal du travail. Si le conseil ne parvient pas à obtenir une entente entre les parties, il doit alors faire une recommandation sur toute la convention collective. Les parties demeurent libres d'accepter ou de rejeter cette recommandation, mais elle doit être publiée, afin que l'opinion publique fasse pression sur l'une ou l'autre des parties pour qu'elle accepte la recommandation. Le projet prévoit même qu'une copie de la recommandation serait envoyée à chacun des membres des conseils d'administration impliqués et également à chacun des salariés visés par la convention ${ }^{27}$.

Les difficultés commencent véritablement en cas de rejet de la recommandation. Un conseil de conciliation - le même selon le premier projet, un autre selon l'exposé du 5 juin - interviendra sur la détermination des services essentiels, si les parties ne parviennent pas à les fixer par entente directe. S'il ne réussit pas à les mettre d'accord, le conseil de conciliation, de nouveau, doit remettre ses recommandations quant aux services essentiels à maintenir, recommandations qui seront également publiées, cette fois encore avec le même objectif de pression sur les parties. Le droit à la grève peut être exercé dès qu'il y a eu entente sur les services essentiels ou que les recommandations du conseil de conciliation à ce sujet ont été publiées ${ }^{28}$.

Ce projet s'inspire très fortement de la proposition syndicale faite au Conseil consultatif à l'automne de 1973. Le ministre du Travail l'admet ouvertement ${ }^{29}$. La philosophie d'une disposition aussi libérale, c'est que les mesures coercitives, en matière de relations de travail, sont inefficaces : si la loi prévoit le recours à un tiers, avec décision exécutoire, il est plus que probable que les parties ne s'entendront pas et remettront l'odieux

27 Les sources de renseignement sur ce projet d'article 99 ont été mentionnés dans les notes 24 et 25 .

28 Ibid., 5 juin 1974, pp. B-2991 et B-2992, et 6 juin 1974, pp. B-3043 et B3044.

29 Ibid., 5 juin 1974, p. B-2993. 
de la décision à cette tierce partie; le fait que la décision vienne d'un tiers incitera même les intéressés à ne s'y conformer que mollement, ou pas du tout.

Le projet a le mérite d'opter pour une position claire, qui mériterait d'être expérimentée. Par contre, les tactiques syndicales des dernières années en amènent plusieurs à douter du bien-fondé d'un tel projet. L'absence de détermination finale par une autorité reconnue, en cas de mésentente entre les parties, fait naître bien des craintes, d'autant plus que le nouveau projet supprime aussi tout recours à l'injonction, comme on peut le faire actuellement en vertu du présent article 99 . La suppression d'un tel recours paraît bien nécessaire, cependant, dans la philosophie choisie : si l'on veut reporter sur les parties la pleine responsabilité de leurs gestes et décisions, tant au niveau du contenu de la négociation que du maintien des services essentiels, il faut supprimer tout refuge dans l'attente d'une intervention extérieure. Une autre critique vient du fait que le nouveau projet limite les services essentiels à ceux «qui doivent être maintenus pour assurer la protection de la santé et de la sécurité » du public, alors que le projet de loi 89 avait ajouté «le bien-être public » à la santé, à la sécurité et à l'éducation; le ministre, dans son projet dont on ne sait pas s'il sera présenté - a fait droit à l'objection syndicale selon laquelle le terme de «bien-être public » pourrait justifier une intervention de l'Etat dans n'importe quel domaine, supprimant ainsi, à toute fin pratique, la garantie du libre exercice du droit de grève ${ }^{30}$.

\section{Opinions des parties}

Pendant que se poursuivaient ces discussions, plus ou moins à huis clos, les principales parties syndicales concernées par les futures négociations faisaient connaître leurs positions respectives. Celles-ci sont loin d'être identiques.

Alors que les chefs parlent, de temps à autre, d'un front commun inter-centrales ${ }^{31}$, les divers groupes s'attachent davantage aux intérêts de chaque secteur. Ainsi, la Fédération des affaires sociales (C.S.N.), qui regroupe de très nombreux syndicats dans les différents domaines de la santé et des services sociaux, a opté carrément, lors de son congrès en mai 1974 , pour un front commun des affaires sociales $\$ 32$. Une réso-

30 Ibid., 6 juin 1974, p. B-3045 et B-3046.

31 La Presse, mercredi 3 juillet 1974, p. E 1 et jeudi 4 juillet 1974, p. A 3.

32 La Presse, jeudi 23 mai 1974, p. A 10. 
lution précisait que, s'il y avait front commun avec d'autres centrales toujours au niveau du seul secteur des affaires sociales - il faudrait respecter le nombre de membres de chaque groupe, ce qui équivaut pratiquement à rejeter un front commun inter-centrales, ou à le soumettre à la domination de la F.A.S. (C.S.N.). A la C.E.Q., on affirme qu'il faudra d'abord «créer un véritable front de l'enseignement ; s'il y a front commun, on devra l'établir à partir d'une solidarité bien exprimée à la base, dans les régions, avant toute action en commun à un niveau plus étendu ${ }^{33}$. Partout on semble craindre de retomber dans les difficultés qu'avait engendrées le front commun de 1972.

Quant au processus de la négociation et du règlement des conflits, la Fédération des affaires sociales (C.S.N.), pour sa part, endosse la proposition du groupe syndical au Conseil consultatif du travail et de la main-d'oeuvre. On insiste, en particulier, pour que les services essentiels à maintenir soient déterminés par libre entente entre les parties, et ce à la base même de la structure, c'est-à-dire entre les parties qui sont nommées au certificat d'accréditation, en l'occurence tel hôpital et tel syndicat local de tel groupe particulier ${ }^{34}$.

On aura remarqué que les discussions rapportées jusqu'ici se sont poursuivies en rapport avec tout le secteur public et parapublic, et que l'accent a toujours été mis sur d'autres secteurs que la fonction publique proprement dite, en particulier sur les hôpitaux et l'éducation. Pour le gouvernement, nous avons vu qu'il se préoccupe d'abord, en vue des modifications au Code du travail, des services publics fournis par un employeur unique, donc des autres services publics que ceux qui tombaient sous le front commun en 1972. Le ministre du Travail a déclaré par ailleurs, que la formule qu'on utiliserait pour les services publics relevant d'entreprises monolithiques devrait inspirer le Conseil consultatif et même l'Assemblée nationale pour le secteur public et parapublic; il insiste "pour qu'on prenne cette nouvelle méthode (et) qu'on l'adapte aux services publics gouvernementaux $\gg 35$.

De son côté, le Syndicat des fonctionnairees provinciaux du Québec vient de présenter (septembre 1974) un document suggérant de nouveaux mécanismes de négociation et de règlement des conflits, différents en

33 La Presse, jeudi 4 juillet 1974, p. A 3.

34 La Presse, jeudi 23 mai 1974, p. A 10.

35 Québec, Assemblée nationale, Journal des Débats, Commission permanente du travail, de la main-d'oeuvre et de l'immigration, 5 juin 1974, p. B-2993. 
bonne partie de ceux que proposent les centrales syndicales ${ }^{36}$. Le S.F.P.Q. prévoit, en même temps, qu'il n'y aura pas de front commun, selon la formule de 1972, mais des contacts et des échanges entre les centrales.

Comme pour les autres groupes syndicaux, le S..F.P.Q. ne veut pas de méthode coercitive dans la détermination du contenu de la convention collective. Selon lui, il ne devrait y avoir de médiation que sur demande volontaire des deux parties; par contre, à la demande d'une des parties, un conseil d'arbitrage serait obligatoirement forrmé et, faute d'entente en cours d'arbitrage, son rapport devrait être publié pour exercer l'influence appropriée sur l'opinion publique, afin que celle-ci incite les parties à régler sans grève ou lock-out. Il n'est pas question, cependant, de sentence exécutoire.

La recommandation du S.F.P.Q. diffère considérablement de celle des centrales quant aux services essentiels. Le S.F.P.Q. estime qu'on devrait créer un comité mixte permanent pour reviser sans cesse la liste des services qu'on considère comme essentiels; à son avis, il est plus facile de procéder à cette détermination dans les périodes calmes qu'aux moments de tension. En fait, la liste n'aurait qu'à être maintenue à jour. L'exercice du droit de grève ou de lock-out serait interdit à moins d'entente sur les services essentiels et sur la façon de les maintenir; faute d'entente, il y aurait décision par le Tribunal du travail. La position du S.F.P.Q. s'explique peut-être par son expérience sur ce point au cours de ses trois négociations. Alors qu'en 1966, on avait reconnu $32 \%$ des employés, fonctionnaires et ouvriers, comme «effectifs essentiels 》, la proportion était tombée à $12 \%$ en 1968 et à $6 \%$ en 1971; dans ce dernier cas, aucun employé n'avait été jugé essentiel dans neuf ministères sur vingt-six ${ }^{37}$.

Devant ces différences de positions, il n'est pas facile de prévoir quelle orientation le législateur prendra. L'absence totale de coercition et le recours à la seule influence de l'opinion publique représentent une option qui offre beaucoup d'attrait aux professionnels des relations de travail. Il n'en va pas nécessairement de même pour l'ensemble de la population ni, vraisemblablement, pour les députés qui doivent voter le projet de loi. Le débat se cristallise habituellement autour des services

36 Un résumé a paru dans La Presse, jeudi 26 septembre 1974, p. A 7.

37 Syndicat des fonctionnaires provinciaux du Québec: Bulletin des membres, 1, 3 (17 octobre 1973), p. 19. Le tableau donne la subdivision des chiffres par ministère. 
essentiels; le ministre peut bien déclarer qu'il a « un certain degré de confiance dans les autorités syndicales », d'autres ne partagent pas son optimisme ${ }^{38}$.

\section{CHAMP DE LA NÉGOCIATION}

Il reste à traiter d'un troisième aspect, qui ne vise que la fonction publique proprement dite, mais dont l'importance est considérable, les matières négociables. Comme au fédéral et dans toutes les autres provinces canadiennes, le champ d'application de la convention collective qui régit les employés directs de l'Etat du Québec est restreint de différentes manières, particulièrement dans les domaines qui sont de la compétence de la Commission de la fonction publique. Un article de la Loi de la fonction publique précise les matières qui peuvent faire l'objet de la négociation ; il se lit comme suit ${ }^{39}$ :

52a. Les fonctionnaires et ouvriers sont régis par les dispositions de la convention collective qui leur sont applicables ... relativement aux sujets suivants :

a) traitement ou rémunération additionnelle ;

b) heures de travail et durée du travail ;

c) congés ;

d) règlement des griefs ;

e) suspension ;

f) congédiement ;

g) appel d'un employé qui se croit lésé par une décision relativement à son classement.

Toutefois, aucune disposition d'une convention collective ne peut porter sur un autre sujet qui, en vertu de la présente loi, relève de la Commission ou du lieutenant-gouverneur en conseil à moins que la Commission n'y ait concouru par règlement et qu'un tel règlement n'ait été approuvé par le lieutenant-gouverneur en conseil.

D'autres articles de la même loi interdisent de traiter de tel ou tel sujet ou limitent la portée de la convention collective dans une matière négociable (art. 52-53). Les principales restrictions, au Québec comme

38 Québec, Assemblée nationale, Journal des Débats, Commission permanente du travail, de la main-d'oeuvre et de l'immigration, 5 juin 1974, p. B-2993.

39 Loi de la fonction publique, Statuts du Québec, 1965 (1e session), chap. 14, art. 52a, ajouté par S.Q. 1969, chap. 14, art. 35 . 
ailleurs, portent sur la classification (art. 21-26) et sur les promotions (art. 34).

\section{Classification et classement}

La classification relève de la compétence de la Commission; la loi est formelle sur ce point; «Le personnel de la fonction publique est classifié suivant la classification établie par la Commission et approuvée par le lieutenant-gouverneur en conseil » (art. 21). Cependant, dès la première convention collective, en 1966, un article établissait le principe de la coopération entre les parties et la Commission de la fonction publique en vue d'établir une nouvelle classification (art. 15.00). Le même article spécifiait que la nouvelle classification devrait respecter certains principes, comme de grouper tous les emplois similaires alors fractionnés en plusieurs classes - le texte comportait même plusieurs exemples - de respecter le taux minimum de l'ancienne classe inférieure et le taux maximum de l'ancienne classe supérieure, etc. (art. 15.01). On avait donc négocié quelques principes directeurs de la future classification.

La nouvelle classification fut approuvée par l'arrêté-en-conseil 185 du 31 janvier 1967. Très tôt, des difficultés sont apparues au niveau du classement, et un grand nombre de plaintes furent logées, démontrant certaines lacunes dans le plan adopté. Aussi, une revision de la classification fut-elle envisagée. Les travaux de revision furent effectués par un comité consultatif composé de huit membres : trois représentants du gouvernement, trois représentants du syndicat, un président et un secrétaire tous deux de la Commission de la fonction publique ${ }^{40}$. Le 1er mars 1972, une entente formelle est intervenue entre le gouvernement du Québec et le S.F.P.Q. concernant l'intégration des fonctionnaires suite à la revision de la classification ; cette intégration devait prendre effet à compter du 12 octobre $19722^{41}$.

La convention collective 1972-1975 établit le principe que «toute modification apportée à la classification... doit faire l'objet d'une consultation des parties » (art. 17.02). Le gouvernement doit de plus

40 Commission de la fonction publique, Rapport annuel 1969, pp. 26 ss. Voir aussi Commission de la fonction publique, La classification du personnel de la fonction publique. Document d'information. Polycopié, mars 1972. Fascicule I : document général, 132 p.; fasc. II : agents de la paix, 23 p.; fasc. III : personnel ouvrier, 23-5 p. 10662.

41 Gazette officielle de Québec, 104, 47A (1er décembre 1972), pp. 10661- 
consulter le syndicat sur les règlements d'intégration, qui doivent prévoir une procédure d'appel jusqu'à l'arbitrage inclusivement. Le protocole d'entente du 1er mars 1972 précise cette procédure d'appel (art. 6). Celle-ci peut toucher tous les aspects du classement : corps, classe, grade, coefficient d'expérience. A la première étape, un comité, composé d'un nombre égal de représentants du Conseil du Trésor et du syndicat étudie l'appel au mérite. Si la décision n'est pas jugée satisfaisante, le syndicat - et non pas le fonctionnaire - peut soumettre le cas pour décision exécutoire à un arbitre choisi par les deux parties. Suite à l'intégration de 1972, il y eut quelque 14,000 appels, dont environ 2,000 ont été portés à l'arbitrage ; près de la moitié des cas d'arbitrage ont été gagnés par le syndicat ; au printemps de 1974, il en restait 600 à régler ${ }^{42}$.

La classification comme telle demeure cependant toujours la responsabilité exclusive de la Commission de la fonction publique. La présente convention collective exige qu'avant toute modification, les parties soient consultées, y compris le syndicat. Lors de la prochaine négociation, celui-ci tentera d'obtenir que toute modification ou nouvelle classification faite à la demande d'une des parties fasse l'objet non seulement d'une consultation, mais d'une entente, avec arbitrage exécutoire en cas d'opposition irréductible ${ }^{43}$.

\section{Avancement et promotion}

En matière d'avancement et de promotion, l'évolution est moins avancée. Tout repose sur la déclaration d'éligibilité, qui vient de la Commission de la fonction publique. La loi dit en effet : "Aucun fonctionnaire... n'est nommé ou promu à moins que, d'après une liste d'éligibilité fournie par la Commission, il ne soit éligible à la fonction à laquelle il est nommé au promu .... (art. 34).

La convention collective présentement en vigueur prévoyait la formation d'un comité conjoint qui devait formuler des recommandations à la Commission de la fonction publique en vue d'un règlement éventuel portant sur le point suivant : en cas d'échec à l'avancement de classe, un candidat pourrait, sur demande, être avisé des motifs de la décision du jury, et il pourrait également en appeler d'une décision qu'il jugerait

42 Québec, ministère de la Fonction publique, Rapport annuel 1972-73, p. 44. Journal S.F.P.Q., 13, 48 (20 avril 1974), p. 11. Québec, Assemblée nationale, Journal des Débats, 2e session, 30e Législature, Commission permanente de la fonction publique, 8 avril 1974 , no 17 , p. B-593.

43 Journal S.F.P.Q., 13, 49 (20 juin 1974), p. 19. 
discriminatoire (art. 20.06). Bien que la question ait été plus d'une fois soulevée au comité des relations professionnelles, rien de concret n'a encore été réalisé sur ce point. À l'occasion de la prochaine négociation, le syndicat se propose d'exiger que l'employeur fasse toujours connaître les motifs d'un refus de changement de grade, et que l'employé puisse contester la décision par voie de grief ${ }^{44}$.

Il faut noter qu'une disposition de la loi, sans doute en vue de protéger le principe du mérite comme base de la nomination et de l'avancement, stipule que la règle de l'ancienneté ne peut jouer que dans le cas des ouvriers, et encore seulement s'il y a compétence égale entre plusieurs candidats (art. 34).

Avec le rôle accru du syndicat et des comités conjoints, on assiste à une érosion progressive des responsabilités de la Commission de la fonction publique. À la limite, elle finira par n'exécuter que ce qui a été conçu et réglementé par le gouvernement seul ou par le gouvernement avec les représentants syndicaux. Les fonctions que remplit présentement la Commission sont indispensables; d'un autre côté, face aux critiques, fondées ou non, dont on trouve un écho dans le rapport du protecteur du citoyen et à la Commission permanente de la fonction publique ${ }^{45}$, on peut se demander si, un jour ou l'autre, la Commission ne finira pas par être intégrée au ministère. Le rôle de surveillance du syndicat en serait alors évidemment accru.

\section{CONCLUSIONS}

Il n'est pas facile de dégager de tout ce qui précède un portrait des prochaines négociations dans la fonction publique au Québec. Même s'il n'y a pas eu de développement spectaculaire au cours des deux dernières années, des changements relativement importants ont pris place.

Sur le plan de l'accréditation, le problème des occasionnels, s'il se règle par la reconnaissance de l'accréditation, pourrait constituer un développement majeur dans l'histoire du syndicat et, davantage encore, dans la protection de ces employés de l'État. Par le truchement des discussions du Comité de relations professionnelles et par les ententes qui en ont découlé en matière d'intégration et de classement des fonc-

44 Ibid., p. 20.

45 Québec, Assemblée nationale, Journal des Débats, 2e session, 30e Législature, Commission permanente de la fonction publique, 9 avril 1974, no 19, pp. B679 à B-691. 
tionnaires, on pourrait assister éventuellement à un important élargissement du champ de la négociation entre le gouvernement et les représentants de ses employés. En somme, la marche du syndicalisme fait sentir ses effets progressivement, dans un effort de rationalisation de plus en plus grande au sein de l'immense organisme fortement bureaucratisé qu'est l'État.

Sur le plan des structures de négociation, la situation est beaucoup plus confuse. Alors que personne ne voudrait revivre ce que certains ont appelé le chaos du front commun de 1972, les prises de position les plus diverses s'énoncent de part et d'autre. Le participant le moins loquace, à l'heure actuelle, et d'emblée le plus important, c'est le gouvernement lui-même. Par la législation qu'il doit introduire pour remplacer l'actuel bill 46, plus encore par les décisions administratives qu'il prendra en cours de route, c'est lui qui décidera, en définitive, de la plus grande centralisation ou d'une certaine décentralisation. Souhaitons qu'il ne sacrifie à la simplification administrative ni ses propres employés, directs et indirects, ni le public consommateur de ses services.

Quant aux mécanismes du processus de négociation et du règlement des conflits, c'est encore le gouvernement, cette fois comme législateur, qui devra trancher entre deux options opposées, peut-être également redoutables, soit un régime qui favorise davantage la négociation - en l'absence de toute mesure coercitive - mais sans définir de protection précise pour le public concerné, soit un système qui inclut de telles protections mais qui favorise d'autant moins le règlement des différends par la voie généralement acceptée de la libre négociation entre les parties intéressées.

\section{Public Service Staff Relations in Quebec}

No major development has taken place in Quebec, since 1972, regarding public service staff relations. Some minor events and important discussions are worth mentioning for they will have a definite impact on future negotiations. It must be recalled that, except on a few points that will be mentioned in due course, public service staff relations in Quebec fall under the general law of labour relations as contained in the Quebec Labour Code, especially in section 99 regarding strikes in public services.

\section{BARGAINING STRUCTURES}

These are eight bargaining units, one of which, subdivided between white and blue collar workers, contains more than $75 \%$ of all public servants (see Table 1). 
The union representing these employees was certified in the Statute itself. The other units comprise special groups such as professionals, teachers employed by the government and peace officiers; these groups were certified by the LieutenantGovernor in Council, on the recommendation of a joint committee. Practically all Quebec public servants are unionized.

Any conflict respecting the bargaining unit is decided, since 1969, by the Labour Court. An important case is now pending concerning all casual workers, white and blue collar workers. Having signed up more than 1000 of them, the union (the «Syndicat des fonctionnaires provinciaux du Québec》 - S.F.P.Q.) has applied, in March 1973, to represent them all; the investigation commissioner dismissed the case, in January 1974, on the ground that he had no jurisdiction, because the Civil Service Commission had withdrawn these employees from the application of the Civil Service Act, and placed them under special regulations. On appeal, the Labour Court ruled that such withdrawal did not deprive these employees from their rights under the Labour Code. The file has been returned to the investigation commissioner to complete his enquiry.

All collective agreements in the Quebec public service will terminate on June 30, 1975, except that of the peace officers (prison guards and the like) which expired June 30, 1974. Bargaining for these employees has been going on since the Spring of 1974, and an agreement has been reached in principle (September 1974) waiting to be ratified by the members. Through a labour relations committee, amendments have been made to the other agreements, on points such as sick leave banks and travel expenses. The government has refused to reopen the agreements on the cost-of-living problem; he agreed to pay earlier than foreseen in the agreement part of a cost-of-living bonus, on a lump-sum basis, scheduled for a later date.

As for the bargaining structure of the next round, all interested unions are favouring sector bargaining rather than common front bargaining at one single table. Even at the sector level, interunion cartels are not guaranteed. Coordinated bargaining is much more likely to occur than any other form centralized bargaining. Greater centralization could come through government decision, either by legislation - present Bill 46 has to be replaced before June 30,1975 - or by pressure on bargaining agents to agree on another unit. There is also the possibility of a multi-tier system, some subjects or general rules being bargained at a central table, other matters at various level sector tables.

\section{IMPASSE RESOLUTION AND THE RIGHT TO STRIKE}

The Civil Service Act contains only two provisions regarding impasse resolution and the right to strike. Peace officers are forbidden to strike, and no other group of government employees may go out on strike unless the essential services to be maintained have been determined by mutual agreement or by decision of the Labour Court. For the rest, the Labour Code applies to civil servants as for all other public service employees.

Common front experience of 1972 had raised forcefully the problem of essential services. To try to solve this problem with general rules, Bill 89 - «An Act to ensure public well-being in case of labour conflict - was introduced 
in the National Assembly in December 1972. It would give the government great powers in public service disputes. Following a decision by a three-person committee that a work stoppage would endanger public health, safety, welfare or education, the Cabinet could, in the case of a government public service, suspend the right to strike, while the dispute was referred to the proper parliamentary committee. A secret vote on the last offers was to be held afterwards by a poll commissioner; if accepted by a majority of employees, these offers would become the collective agreement; otherwise the government would then remove the strike prohibition or refer the dispute to the National Assembly for settlement. In non-government public services, the same procedure could be followed, or the case could be referred to «commissioners on disputes》 for a last-offer-selection type of arbitration. The reaction from all sides was so bad that the Minister of Labour finally withdrew the Bill.

In July 1974, Bill 24 was tabled containing many amendments to various parts of the Labour Code, but nothing on section 99. The Advisory Council on Labour and Manpower had discussed possible formulas for impasse resolution without coming to a consensus, mainly on essential services. The union nembers of the Council advocated mediation board intervention, on the essential services to be maintained, if the parties could not agree; the board recommendations would be published to enlist public pressure on the parties, but there would be no compulsory decision. The management members considered such decision by an outside body necessary for the protection of the public.

The Labour Minister has endorsed publicly the no-coercion position, both on the determination of essential services and on the content of the collective agreement. He underlined that compulsory intervention with binding decision, brings no solution to labour disputes; it leads the parties to refuse to shoulder their responsibilities and often induces them to reject such decisions. It is not clear however how much support the Minister will be able to secure on such a position in his own party caucus.

The civil servants union has come up with different recommendations. It suggests that mediation services should be granted only on joint application, but that arbitration should be available at the request of one party; the arbitration award should be made public but it would not be binding. On the question of essential services the S.F.P.Q. considers that a list should be maintained up-todate by a joint permanent committee; in case of a strike, failing agreement of the parties on the list, a decision should be made by the Labour Court.

What will finally come out of such conflicting opinions is hard to know. The no-coercion approach is appealing to labour experts; the apparent security of a legal way to determine at least essential services may be more palatable to the public in general and probably to most of the National Assembly members.

\section{SCOPE OF BARGAINING}

As in all other jurisdictions, scope of bargaining for public servants is restricted in Quebec mainly on questions that fall in the realm of the Civil Service Commission, especially on classification and promotion. 
Classification is the sole responsibility of the Commission, but the very first collective agreement, in 1966, stated that the forthcoming new classification would abide by certain principles, some of them were spelled out in the agreement. The new classification was approved in 1967, but difficulties grew out immediately. A consultative committee for its revision was soon in operation; it was made up of government, union and Commission representatives. Following this revision, the government and the union signed a formal agreement determining the process for the integration of civil servants into the new classification; an appeal procedure was included, with binding arbitration as the final step. 14,000 cases were appealed, and 2,000 submitted to arbitration, nearly half of which were won by the union. In its next round of bargaining, the union plans to go after joint decision on any modification or new classification.

According to the current agreement, a joint committee was to make recommendations on rules that would permit an unsuccessful candidate to a higher class to be informed of the reasons of his failure, and to have the right to appeal any discriminatory decision. No progress has been made on this point, and the union will try to have these rights written out in the next agreement.

To protect the merit principle, seniority cannot be considered for appointment or promotion of a white collar civil servant; for blue collar morkers, it can be used only in cases of equal competence among two or more candidates.

With the progressive encroachment of the collective agreement and joint committee activities on its various responsibilities, the Civil Service Commission progressively loses its decision powers and looks more and more as an administrative arm of the Civil Service Department; it might eventually become simply a division of the Department, the agreement and the union taking up the duties the Commission had to protect the civil servant against arbitrary decisions.

\section{CONCLUSIONS}

In trying to extend the scope of bargaining and to obtain more accurate definitions of the bargaining units - as in the case of casual workers - the civil servants unions carry on the work of bringing greater rationalization in the whole structure and general rules of the Civil Service itself.

The future bargaining structures are uncertain. No one wishes to reenact the chaotic situation of the 1972 common front. But the most important actor, the government, remains silent for the time being concerning its intentions.

The impasse resolution system is in the process of being changed. Conflicting opinions on very basic approaches appear to block any proposal, at this point, for revision of the all-important section 99 of the Labour Code. 